\title{
Interactive comment on "Applying FP_ILM to the retrieval of geometry-dependent effective Lambertian equivalent reflectivity (GE_LER) to account for BRDF effects on UVN satellite measurements of trace gases, clouds and aerosols” by Diego G. Loyola et al.
}

Anonymous Referee \#2

Received and published: 22 July 2019

This manuscript presents a new approach to derive effective scene albedo on a pixelper-pixel basis from TROPOMI observations and to build a viewing zenith angle dependent LER climatology with an improved spatial resolution compared to former data bases. Although the topic of the study fits well within AMT and there is no obvious issue with the approach, I would suggest to further discuss the results and to extend the comparisons to better demonstrate the added-value of the database. For example, results are discussed for only one spectral region and a limited amount of data (April 
2018). It would be beneficial to have more illustrations for different months. Reading the manuscript, I had many comments similar to those from reviewer 1. I won't list those again but encourage the authors to carefully reply to them. Below are a few additional comments. Once the comments have been addressed and the manuscript consolidated, this work will be worth being published within AMT.

\section{Comments:}

- The description of the smart sampling and machine learning approaches is quite technical. It would be beneficial to the readers to further describe the general ideas/concepts on which rely those methods.

- Section 3: Could you provide more details here on how clear-sky pixels are selected? Such details are given later in the manuscript but it would good to already describe this in section 3. Could you also provide some statistics on the number of days required to have a global coverage? There must be some regions with persistent clouds for which the update frequency drastically decreases. Actually, it would be useful for traceability to provide this information in the database along with the G3_LER values. For example, for one given cell, the LER value has been derived from day-1, $-2,-3$, or ...

- G3_LER data seems to be available only for the ozone fitting window and for three surface types. Could you comment why only those three surface have been considered? In other regions than UV, BRDFs effects will differ much more significantly as a function of the surface type. Could you clarify if you intend to provide GLER data in other spectral ranges and how you intend to proceed with respect to this aspect.

- It is mentioned that the Bodeker ozone database is combined with the McPeters/Labow climatology as input of the RT simulations. Could you be more specific on the needs for this combination and on what is provided by each of 
those databases. Also in Table 1, the ozone profiles appear to be classified only as a function of the total column. Is it sufficient or are the geographic variations of the profiles accounted for somehow? Is there any latitude/longitude dependence taken into account? If not, please be more specific on the profiles that have been used. Also could you provide typical sampling steps of the different dimensions?

- Figure 7: to better illustrate the possible impact of BRDF, could you show such clear-sky histograms for different range of viewing angles. If BRDF effect is important, we could expect systematic biases varying as a function of the VZA. Also, biases are more important for cloud cases. Is it because cloud albedo are retrieved in a different spectral region?

- Figure 8 : what are the implications of the numerical instability of the RT simulations around $V Z A=0$ on the retrieved LER?

- Figure 9: There is a clear general bias between the G3_LER and OMI_LER data, even at low/mid-latitudes. Could you better quantify and discuss this? Is there any indication that one of the two data sets would be more realistic?

\section{Minor/Technical comments:}

- Quality of figures is generally low. Could you increase the quality as well as the size of labels?

- Page 2 - line 6: 35\% on ozone column seems large. Is this value correct?

- Page 2 - line 12: Could you be more specific with that statement? Are there some references providing estimates of errors on TROPOMI products caused by the too coarse resolution of old databases?

Printer-friendly version

Interactive

comment

Page 3 - line 29: Add "solar and" before "viewing geometry"? 
- Page 6 - line 8: The LER data could still differ from the actual surface properties in case of sudden snow fall combined with significant cloudiness.

AMTD

- Page 6 - line 16: remove "viewing geometry"

- Figure 4 shows negative optical densities, which is not physical. In the text, those quantities are referred to optical densities differences but it is not clear what is the reference. Could you homogenize the text and y-label and clarify what are those optical density differences?

- Page 7 - lines 5-6: This is very technical and the meaning is not clear at all for me. Could you rephrase this?

- Page 8 - line 2: "from the couple of days" is not clear. Please be more specific.

- Page 8 - line 29: "smoother" instead of "smother"

- Page 9 - line 31: Mention that those numbers are valid for April 2018.

Interactive comment on Atmos. Meas. Tech. Discuss., doi:10.5194/amt-2019-37, 2019. 